\title{
THE STRUCTURE OF THE RICCI TENSOR ON LOCALLY HOMOGENEOUS LORENTZIAN GRADIENT RICCI SOLITONS
}

\author{
M. BROZOS-VÁZQUEZ E. GARCÍA-RÍO S. GAVINO-FERNÁNDEZ P. GILKEY
}

\begin{abstract}
We describe the structure of the Ricci tensor on a locally homogeneous Lorentzian gradient Ricci soliton. In the non-steady case, we show the soliton is rigid in dimensions three and four. In the steady case, we give a complete classification in dimension three.
\end{abstract}

\section{INTRODUCTION}

Let $(M, g)$ be a Lorentzian manifold of dimension $n+2$ for $n \geq 1$, let $\rho$ be the Ricci tensor. Let Ric be the Ricci operator; $\rho(X, Y)=g(\operatorname{Ric} X, Y)$. If $f \in C^{\infty}(M)$, let Hess $f$ be the Hessian; $f$ is often called the potential function. Then

$$
\operatorname{Hess}_{f}(X, Y)=\left(\nabla_{X} d f\right)(Y)=X Y(f)-\left(\nabla_{X} Y\right)(f) .
$$

Let $\nabla f$ be the vector field dual to the exterior derivative $d f$ of $f$; this will also be denoted by grad $\{f\}$ for notational clarity when convenient. The Hessian operator

$$
\mathcal{H}_{f}(X):=\nabla_{X}(\nabla f) \text { satisfies } \operatorname{Hess}(X, Y)=g\left(\mathcal{H}_{f} X, Y\right) \text {. }
$$

Note that $\|\rho\|^{2}=\|\operatorname{Ric}\|^{2}$ and $\left\|\mathcal{H}_{f}\right\|^{2}=\left\|\operatorname{Hess}_{f}\right\|^{2}$.

The triple $(M, g, f)$ is said to be a Lorentzian gradient Ricci soliton if $f$ satisfies the gradient Ricci soliton equation:

$$
\operatorname{Hess}_{f}+\rho=\lambda g \text { for some } \lambda \in \mathbb{R} \text {. }
$$

Setting $f=0$ yields the Einstein equation $\rho=\lambda g$; thus Equation (1) is a natural generalization of the Einstein equation and a gradient Ricci soliton can be thought of as a generalized Einstein manifold. Gradient Ricci solitons also correspond to self-similar solutions of the Ricci flow $\partial_{t} g(t)=-2 \rho_{g(t)}$. For these reasons, gradient Ricci solitons have been extensively investigated in the literature - see for example the discussion in [4, 10, 13, 23, and the references therein. If $\lambda>0$ (resp. $\lambda=0$ or $\lambda<0)$, then $(M, g, f)$ is said to be shrinking (resp. steady or expanding). We shall assume for the most part that $(M, g)$ is locally homogeneous. This implies the scalar curvature is constant.

One has canonical examples which play a central role in the theory. Let $\left(N, g_{N}\right)$ be an Einstein manifold with Einstein constant $\lambda$, i.e. $\rho_{N}=\lambda g_{N}$. Let $M=N \times \mathbb{R}^{k}$ have the product metric $g_{M}$ and let $f(x):=\frac{\lambda}{2}\|\pi(x)\|^{2}$ where $\pi$ is projection on the second factor. Then $\left(M, g_{M}, f\right)$ is a gradient Ricci soliton and is said to be rigid.

2010 Mathematics Subject Classification. 53C21, 53B30, 53C24, 53C44.

Key words and phrases. Bochner identity, Cahen-Wallach space, gradient Ricci soliton, harmonic Weyl tensor, Hessian, Killing vector field, locally homogeneous Lorentzian manifold, Walker manifold.

Partially supported by projects EM2014/009, GRC2013-045 and MTM2013-41335-P with FEDER funds (Spain). 
Since we are interested in questions of local geometry, by an abuse of notation we shall also say that $\left(M, g_{M}, f\right)$ is rigid if $\left(M, g_{M}, f\right)$ is isomorphic to an open subset of a product $N \times \mathbb{R}^{k}$ which is rigid. We shall use the following results of Petersen and Wylie 24. Assertion (2) was first proved in the Riemannian setting but extends easily to arbitrary signature.

Theorem 1. 24

(1) Any locally homogeneous Riemannian gradient Ricci soliton is rigid.

(2) Let $(M, g)=\left(M_{1} \times M_{2}, g_{1} \oplus g_{2}\right)$ be the direct product of two pseudoRiemannian manifolds. If $f$ satisfies the gradient Ricci soliton equation on $(M, g)$, then $f\left(x_{1}+x_{2}\right)=f_{1}\left(x_{1}\right)+f_{2}\left(x_{2}\right)$ where $f_{1}$ and $f_{2}$ satisfy the gradient Ricci soliton equation on $\left(M_{1}, g_{1}\right)$ and on $\left(M_{2}, g_{2}\right)$ separately.

Assertion (1) was originally proven for homogeneous manifolds, but the assumption of homogeneity can be weakened to local homogeneity by modifying the argument in 24] Proposition 1 as in the proof of Lemma 2(2c). Since any locally homogeneous Riemannian gradient Ricci soliton is rigid, the classification is complete in this context. However the possible geometries are much richer in the Lorentzian setting owing to the existence of degenerate parallel line fields. For example, in Example 17 we shall present results of [1] showing that Cahen-Wallach symmetric spaces admit steady non-rigid gradient Ricci solitons.

1.1. Outline of the paper and summary of results. In Section 1.2 , we state Lemma 2. This Lemma, which will be proved in Section 2 summarizes the relevant results we shall need concerning gradient Ricci solitons with constant scalar curvature; many of these results rely upon earlier papers. The analysis there will be local in nature and will rely on the investigation of the gradient Ricci soliton Equation (11) as this links the geometry of the manifold, through its Ricci curvature, with the extrinsic geometry of the level sets of the potential function by means of their second fundamental form. The signature of the manifold plays no role in Lemma 2 and is completely general. We shall see that if the scalar curvature is constant, then any solution of (11) is an isoparametric function, i.e.

$$
\|\nabla f\|^{2}=b(f) \text { and } \Delta f=a(f) \text { for } a, b \text { smooth on Range }(f) .
$$

For the remainder of the paper we shall assume (unless otherwise noted) that the underlying manifold $(M, g)$ is a locally homogeneous Lorentzian manifold and that $(M, g, f)$ is a gradient Ricci soliton. In Section 1.3 we present our results in

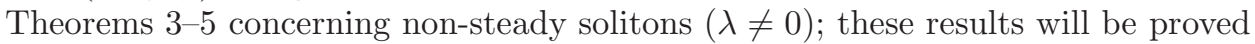
in Section 3. In low dimensions, such solitons are rigid; in arbitrary dimensions, the eigenvalue structure of the Ricci operator agrees with the corresponding eigenvalue structure of a rigid soliton, i.e. there are only two eigenvalues $\{0, \lambda\}$. In Section 1.4 we present our results concerning steady solitons $(\lambda=0)$ in Theorems 8 , these will be proved in Section 4 . Theorem 8 gives a complete classification if $\|\nabla f\|^{2}<$ 0 . In Theorem 9, we shall examine the situation when $\|\nabla f\|^{2}=0$ and show the Ricci tensor is either 2 or 3 step nilpotent; the metrics in question are pure radiation metrics with parallel rays [21. If we further restrict the geometry, stronger results are available. In Section 1.5 we give a complete classification of symmetric Lorentzian gradient Ricci solitons in Theorem 12 This result will be proved in 
Section 5. In Section [1.6 in Theorem 16] we give a complete classification of 3dimensional Lorentzian locally homogeneous gradient Ricci solitons; there are 3 non-trivial families of examples. Theorem 16 will be proved in Section 6.

The fact that $(M, g)$ is Lorentzian plays a crucial role in many arguments. For example, when we study the non-steady case, there exists a distinguished null parallel vector field and there do not exist orthogonal null vector fields - this is a Lorentzian phenomena not present in the Riemannian or the higher signature setting. The fact that $(M, g)$ is locally homogeneous is not simply used to ensure that the scalar curvature is constant, it plays a role in many proofs where we take frame fields consisting at least in part of Killing vector fields. As our discussion is local in nature, it is not necessary to impose global conditions such as global homogeneity or completeness.

1.2. Consequences of the gradient Ricci soliton equation. Let $\tau$ be the scalar curvature. Let $\nabla f$ be the vector field which is dual to the 1 -form $d f$. It is characterized by the identity

$$
g(\nabla f, X)=X(f) \text { for any vector field } X .
$$

Let $\mathcal{L}$ be the Lie derivative; a vector field $X$ on $(M, g)$ is Killing if $\mathcal{L}_{X} g=0 ; X$ is Killing if and only if

$$
g\left(\nabla_{X} Z, X\right)=0 \text { for any vector field } X .
$$

We say $(M, g, f)$ is isotropic if $\|\nabla f\|^{2}=0$. Section 2 is devoted to the proof of the following quite general result concerning gradient Ricci solitons with constant scalar curvature in arbitrary signature.

Lemma 2. Let $(M, g, f)$ be a gradient Ricci soliton with constant scalar curvature.

(1) We have the following relations:

(a) $\operatorname{Ric}(\nabla f)=0$.

(b) $\|\nabla f\|^{2}-2 \lambda f=$ const.

(c) $R(X, Y, Z, \nabla f)=\left(\nabla_{X} \rho\right)(Y, Z)-\left(\nabla_{Y} \rho\right)(X, Z)$.

(d) $\left(\nabla_{\nabla f}\right.$ Ric $)+\operatorname{Ric} \circ \mathcal{H}_{f}=R(\nabla f, \cdot) \nabla f$.

(2) Let $X$ be a Killing vector field.

(a) $\mathcal{L}_{X}\left(\operatorname{Hess}_{f}\right)=\operatorname{Hess}_{X(f)}$.

(b) $\operatorname{grad}\{X(f)\}$ is a parallel vector field.

(c) If $\lambda \neq 0$, then $\operatorname{grad}\{X(f)\}=0$ if and only if $X(f)=0$.

(3) $\lambda((n+2) \lambda-\tau)=\left\|\operatorname{Hess}_{f}\right\|^{2}$.

(4) If $(M, g, f)$ is isotropic and non-steady, then $(M, g)$ is Einstein.

(5) If $(M, g, f)$ is steady, then $\|$ Hess $f \|^{2}=0$ and $\|\nabla f\|^{2}=\mu$ is constant.

We shall apply different techniques in what follows to study the steady and the non-steady cases since setting $\lambda \neq 0$ or $\lambda=0$ in Lemma2 2 gives significantly different information about the potential function $f$. By Lemma 2, any isotropic non-steady gradient Ricci soliton with constant scalar curvature is Einstein. However, there exist isotropic steady gradient Ricci solitons which are not Einstein [1.

1.3. Non-steady locally homogeneous Lorentzian gradient Ricci solitons. We say that a Lorentzian manifold $(M, g)$ is irreducible if the holonomy representation has no non-trivial invariant subspace and that $(M, g)$ is indecomposable if the metric on any non-trivial subspace fixed by the holonomy representation is degenerate and thus the holonomy representation does not decompose as a non-trivial 
direct sum of subrepresentations. The distinction between irreducible and indecomposable is only relevant in the indefinite setting. We shall establish the following results in Section 3

Theorem 3. Let $(M, g, f)$ be a locally homogeneous Lorentzian non-steady gradient Ricci soliton. Then one of the following holds:

(1) $(M, g)$ is irreducible and Einstein.

(2) $(M, g, f)$ is rigid, this is, there is a local splitting $(M, g, f)=\left(N \times \mathbb{R}_{\nu}^{k}, g_{N}+\right.$ $\left.g_{e}, f_{N}+f_{e}\right)$ where $\left(N, g_{N}\right)$ is Einstein with Einstein constant $\lambda$ and $\left(\mathbb{R}_{\nu}^{k}, g_{e}, f_{e}\right)$ is pseudo-Euclidean space, $\nu=0,1$, with $f_{e}(x):=\frac{\lambda}{2}\|x\|^{2}$.

(3) $(M, g, f)$ locally splits as

$$
(M, g, f)=\left(N_{0} \times N_{1} \times \mathbb{R}^{k}, g_{0}+g_{1}+g_{e}, f_{0}+f_{1}+f_{e}\right)
$$

where $\left(N_{0}, g_{0}, f_{0}\right)$ is an indecomposable locally homogeneous Lorentzian gradient Ricci soliton, $\left(N_{1}, g_{1}\right)$ is a Riemannian Einstein manifold with Einstein constant $\lambda$ and $\left(\mathbb{R}^{k}, g_{e}, f_{e}\right)$ is Euclidean space with $f_{e}(x):=\frac{\lambda}{2}\|x\|^{2}$.

We now focus on the situation in Assertion (3) above and study the indecomposable factor. Recall that a Lorentzian manifold is said to be Walker if it admits a parallel null line field, and strict Walker if this distribution is spanned by a parallel null vector field; we refer to [6] for further details. We shall say that $(M, g)$ has harmonic Weyl tensor if the Schouten tensor $S$ is Codazzi. This means (see [2]):

$$
\nabla_{X} S_{Y Z}=\nabla_{Y} S_{X Z} \text { where } S=\rho-\frac{\tau}{2(n+1)} g .
$$

Theorem 4. Let $(M, g, f)$ be a locally homogeneous indecomposable Lorentzian non-steady gradient Ricci soliton which is not Einstein.

(1) Locally, there exists a Killing vector field $X$ so $U:=\operatorname{grad}\{X(f)\}$ is a non-trivial parallel null vector field; thus $(M, g)$ is strict Walker.

(2) $U$ is unique up to scale, $\mathcal{V}:=\{U, \nabla f\} \subset \operatorname{ker}\{\mathrm{Ric}\}$ is a $U$-parallel Lorentzian distribution, and grad $\{U(f)\}=\lambda U$.

(3) $\nabla_{U}$ Ric $=\nabla_{U} \mathcal{H}_{f}=0$, Spec $\{$ Ric $\}=\operatorname{Spec}\left\{\mathcal{H}_{f}\right\}=\{0, \lambda\}$, Ric and $\mathcal{H}_{f}$ are diagonalizable, $\operatorname{ker}\{\operatorname{Ric}\}=\operatorname{Image}\left\{\mathcal{H}_{f}\right\}$, and $\operatorname{ker}\left\{\mathcal{H}_{f}\right\}=\operatorname{Image}\{\operatorname{Ric}\}$.

(4) The Weyl tensor of $(M, g)$ is harmonic if and only if $(M, g, f)$ is rigid.

(5) If $\operatorname{dim}(\operatorname{ker}\{\operatorname{Ric}\})=2$, then $(M, g, f)$ is rigid.

This leads to the following classification result in low dimensions:

Theorem 5. Let $(M, g, f)$ be a locally homogeneous Lorentzian non-steady gradient Ricci soliton of dimension $m \leq 4$. Then $(M, g, f)$ is rigid.

Remark 6. What is indeed proven in Theorem 5 is that if the factor $N_{0}$ of the decomposition given in Theorem 3 above is of dimension $n_{0} \leq 4$ then the gradient Ricci soliton is rigid.

1.4. Steady locally homogeneous Lorentzian gradient Ricci solitons. The geometry of the level sets of the potential function plays an essential role in our analysis; the norm $\|\nabla f\|^{2}$ is important as this controls the nature of the metric on the level sets. The 2-dimensional case is trivial; one has [4, 14]:

Theorem 7. A steady locally homogeneous Ricci soliton of dimension 2 either in the Riemannian or in the Lorentzian setting is flat. 
The following two results will be established in Section 4

Theorem 8. Let $(M, g, f)$ be a locally homogeneous steady gradient Lorentzian Ricci soliton. If $\|\nabla f\|^{2}<0$, then $(M, g)$ splits locally as an isometric product $\left(\mathbb{R} \times N,-d t^{2}+g_{N}\right)$, where $\left(N, g_{N}\right)$ is a flat Riemannian manifold and $f$ is orthogonal projection on $\mathbb{R}$.

The cases when $\|\nabla f\|^{2} \geq 0$ are less rigid in the steady setting. Several examples in the spacelike case $\|\nabla f\|^{2}>0$ are known 1, 4, but little more of a general nature is known about this case. In the isotropic case one has some restrictions on the Ricci operator; in particular, it must be nilpotent. Recall that a tensor $T$ is said to be recurrent if there is a smooth 1 -form $\omega$ so that $\nabla_{X} T=\omega(X) T$.

Theorem 9. Let $(M, g, f)$ be an isotropic locally homogeneous Lorentzian steady gradient Ricci soliton. One of the following two possibilities pertains:

(1) $\mathcal{H}_{f}=-$ Ric has rank 2 and is 3-step nilpotent.

(2) $\mathcal{H}_{f}=-$ Ric has rank 1 and is 2-step nilpotent. In this case $(M, g)$ is locally a strict Walker manifold, more specifically:

(a) $\operatorname{ker}\left\{\mathcal{H}_{f}\right\}=\nabla f^{\perp}$ and Image $\left\{\mathcal{H}_{f}\right\}=\nabla f$.

(b) $\nabla f$ is a recurrent vector field and $\nabla f^{\perp}$ is an integrable totally geodesic distribution with leaves the level sets of $f$.

(c) Let $P \in M$. At least one of the following possibilities holds near $P$.

(i) There exists a Killing vector field $F$ so $\operatorname{grad}\{F(f)\}$ is a null parallel vector field.

(ii) There exists a smooth function $\psi$ defined near $P$ so $\psi \nabla f$ is a null parallel vector field.

We shall illustrate possibility (2) in Example 17 presently.

1.5. Symmetric Lorentzian gradient Ricci solitons. Stronger results are available if $(M, g)$ is locally symmetric; this implies $\nabla R=0$.

Definition 10. We say that $\left(N, g_{N}\right)$ is a Cahen-Wallach symmetric space if there are coordinates $\left(t, y, x_{1}, \ldots, x_{n}\right)$ so:

$$
g=2 d t d y+\left(\sum_{i=1}^{n} \kappa_{i} x_{i}^{2}\right) d y^{2}+\sum_{i=1}^{n} d x_{i}^{2} \text { for } 0 \neq \kappa_{i} \in \mathbb{R} .
$$

We shall always assume that all $\kappa_{i} \neq 0$ to ensure that $\left(N, g_{N}\right)$ is indecomposable.

We refer to [7, 8] for the proof of Assertion (1) in the following result and to [1] for the proof of Assertion (2) in the following result:

\section{Theorem 11.}

(1) Let $(M, g)$ be a Lorentzian locally symmetric space.

(a) If $(M, g)$ is irreducible, then $(M, g)$ has constant sectional curvature.

(b) If $(M, g)$ is indecomposable but reducible, then $(M, g)$ is a CahenWallach symmetric space.

(2) If $(M, g, f)$ is a Cahen-Wallach gradient Ricci soliton, then $(M, g, f)$ is steady, $f=a_{0}+a_{1} y+\frac{1}{4} \sum_{i} \kappa_{i} y^{2}$, and $\nabla f=\left(a_{0}+\frac{1}{2} \sum_{i} \kappa_{i} y\right) \partial_{t}$ is null.

Theorem 11 will play a crucial role in the proof that we shall give of the following result in Section 5 . 
Theorem 12. Let $(M, g, f)$ be a locally symmetric Lorentzian gradient Ricci soliton. Then $(M, g)$ splits locally as a product $M=N \times \mathbb{R}^{k}$ where

(1) if $(M, g, f)$ is not steady, then $\left(N, g_{N}\right)$ is Einstein and the soliton is rigid,

(2) if $(M, g, f)$ is steady, then $\left(N, g_{N}, f_{N}\right)$ is locally isometric to a CahenWallach symmetric space.

1.6. Three-dimensional locally homogeneous gradient Ricci solitons. We will establish the following 2 results in 3-dimensional geometry in Section [6 Let $(M, g)$ be a Lorentzian manifold of dimension 3. We suppose first that $(M, g)$ is strict Walker, i.e. admits a null parallel vector field. We may then (see, for example, [6]) find local adapted coordinates $(t, x, y)$ so that

$$
g=2 d t d y+d x^{2}+\phi(x, y) d y^{2} .
$$

The following is of independent interest; we drop for the moment the assumption that the metric is locally homogeneous and focus on Walker geometry:

Theorem 13. Let $(M, g)$ be a non-flat 3-dimensional Lorentzian strict Walker manifold. Then $(M, g, f)$ is a gradient Ricci soliton if and only if there exist a cover of $M$ by coordinate systems where the metric has the form given in Equation (5) where one of the following occurs:

(1) $\phi(x, y)=\frac{1}{\alpha^{2}} a(y) e^{\alpha x}+x b(y)+c(y)$ and $f(x, y)=x \alpha+\gamma(y)$ where $\alpha \in \mathbb{R}$ and $\gamma^{\prime \prime}(y)=-\frac{1}{2} \alpha b(y)$. In this setting, $\nabla f=\alpha \partial_{x}+\gamma^{\prime}(y) \partial_{t}$ is spacelike.

(2) $\phi(x, y)=x^{2} a(y)+x b(y)+c(y)$ and $f(x, y)=\gamma(y)$ where $\gamma^{\prime \prime}(y)=\frac{1}{4} a(y)$. In this setting $\nabla f=\gamma^{\prime} \partial_{t}$ is null.

Moreover, in both cases the Ricci soliton is steady.

Definition 14. Adopt the notation of Equation (5).

(1) Let $\phi(x, y)=b^{-2} e^{b x}$ for $0 \neq b \in \mathbb{R}$ define $\mathcal{N}_{b}$.

(2) Let $\phi(x, y)=\frac{1}{2} x^{2} \alpha(y)$ where $\alpha_{y}(y)=c \alpha^{3 / 2}(y)$ and $\alpha(y)>0$ define $\mathcal{P}_{c}$.

(3) Let $\phi(x, y)= \pm x^{2}$ define the Cahen-Wallach symmetric space $\mathcal{C} \mathcal{W}_{ \pm}$.

The following result was established in [17]:

Theorem 15. Let $(M, g)$ be a locally homogeneous Lorentzian strict Walker manifold of dimension 3 . Then $(M, g)$ is locally isometric to one of the manifolds given in Definition 14.

We can now state our classification result:

Theorem 16. Let $(M, g, f)$ be a Lorentzian locally homogeneous gradient Ricci soliton of dimension 3 . If $(M, g, f)$ is non-trivial, then either it is rigid or $(M, g)$ is locally isometric to either $\mathcal{C W}_{ \pm}, \mathcal{P}_{c}$ or $\mathcal{N}_{b}$ as defined above and the soliton is steady. Moreover $\nabla f$ is null if $(M, g)=\mathcal{P}_{c}$ or if $(M, g)=\mathcal{C W}_{ \pm}$, and $\nabla f$ is spacelike if $(M, g)=\mathcal{N}_{b}$.

\section{Consequences of the gradient Ricci soliton equation The Proof of Lemma 2}

In Section 2.1 we establish Assertion (1), in Section 2.2 we derive Assertion (2), in Section 2.3, we prove Assertion (3), in Section 2.4. we verify Assertion (4), and in Section 2.5 we complete the proof of Lemma 2 by checking Assertion (5). 
2.1. The proof of Lemma 2 (1). If $(M, g, f)$ is a gradient Ricci soliton, then $\nabla \tau=2 \operatorname{Ric}(\nabla f)$ [15, 25. Assertion (1a) now follows as $\nabla \tau=0$. We also have 4, 13, 15, 25] that $\tau+\|\nabla f\|^{2}-2 \lambda f=$ const; Assertion (1b) now follows. We refer to [4, 16 for the proof of Assertion (1c) which holds without assuming $\tau=$ const. The identity

$$
\left(\nabla_{\nabla f} \text { Ric }\right)+\operatorname{Ric} \circ \mathcal{H}_{f}=R(\nabla f, \cdot) \nabla f+\frac{1}{2} \nabla \nabla \tau
$$

was proved in the Riemannian setting [25. One can use analytic continuation to extend this identity to the indefinite setting (or simply observe the proof goes through without change in the higher signature context). Assertion (1d) now follows once again using the fact that $\tau$ is constant.

2.2. The proof of Lemma 2 (2). Let $X$ be a Killing vector field. Fix a point $P$ of $M$ so that $X(P) \neq 0$; Assertion (2) for $P$ where $X(P)=0$ will then follow by continuity. Choose a system of local coordinates $\left(x_{1}, \ldots, x_{n+2}\right)$ so that $X=\partial_{x_{1}}$. Set $g_{i j}:=g\left(\partial_{x_{i}}, \partial_{x_{j}}\right)$ and observe that

$$
\begin{aligned}
\partial_{x_{1}} g_{i j} & =g\left(\nabla_{\partial_{x_{1}}} \partial_{x_{i}}, \partial_{x_{j}}\right)+g\left(\partial_{x_{i}}, \nabla_{\partial_{x_{1}}} \partial_{x_{j}}\right) \\
& =g\left(\nabla_{\partial_{x_{i}}} \partial_{x_{1}}, \partial_{x_{j}}\right)+g\left(\partial_{x_{i}}, \nabla_{\partial_{x_{j}}} \partial_{x_{1}}\right)=\left(\mathcal{L}_{\partial_{x_{1}}} g\right)\left(\partial_{x_{i}}, \partial_{x_{j}}\right) .
\end{aligned}
$$

Thus $\partial_{x_{1}} g_{i j}=0$ so $\partial_{x_{1}} \Gamma_{i j}{ }^{k}=0$ as well. We establish Assertion (2a) by computing:

$$
\begin{aligned}
& \left(\mathcal{L}_{\partial_{x_{1}}} \operatorname{Hess}_{f}\right)\left(\partial_{x_{i}}, \partial_{x_{j}}\right)=\mathcal{L}_{\partial_{x_{1}}} \operatorname{Hess}_{f}\left(\partial_{x_{i}}, \partial_{x_{j}}\right) \\
= & \mathcal{L}_{\partial_{x_{1}}}\left(\partial_{x_{i} x_{j}}^{2}(f)-\Gamma_{i j}{ }^{k} \partial_{x_{k}}(f)\right) \\
= & \partial_{x_{1} x_{i} x_{j}}^{3}(f)-\partial_{x_{1}}\left(\Gamma_{i j}{ }^{k}\right) \partial_{x_{k}}(f)-\Gamma_{i j}{ }^{k} \partial_{x_{1} x_{k}}^{2}(f) \\
= & \partial_{x_{i} x_{j}}^{2} \partial_{x_{1}}(f)-\Gamma_{i j}{ }^{k} \partial_{x_{k}} \partial_{x_{1}}(f)=\operatorname{Hess}_{\partial_{x_{1}}(f)}\left(\partial_{x_{i}}, \partial_{x_{j}}\right) .
\end{aligned}
$$

Since $\mathcal{L}_{X} g=0$ and since $\rho$ is natural, $\mathcal{L}_{X} \rho=0$. Equation (1) implies that $\mathcal{L}_{X} \operatorname{Hess}_{f}=0$, and therefore by Assertion (2a), $\operatorname{Hess}_{X(f)}=0$. Consequently, $\operatorname{grad}\{X(f)\}$ is parallel. This establishes Assertion (2b). Assume now that $\lambda \neq 0$. It is clear that $\operatorname{grad}\{X(f)\}=0$ if $X(f)=0$. Conversely, if $\operatorname{grad}\{X(f)\}=0$, then $X(f)=\kappa$ for some constant $\kappa$. Since the scalar curvature is constant, Assertion (1) implies that $\operatorname{Ric}(\nabla f)=0$. Since $X$ is a Killing vector field,

$$
\begin{aligned}
0 & =\nabla f(\kappa)=\nabla f(X(f))=\nabla f g(\nabla f, X)=g\left(\nabla_{\nabla f} \nabla f, X\right)+g\left(\nabla f, \nabla_{\nabla f} X\right) \\
& =\operatorname{Hess}_{f}(\nabla f, X)+\frac{1}{2}\left(\mathcal{L}_{X} g\right)(\nabla f, \nabla f)=-\rho(\nabla f, X)+\lambda g(\nabla f, X)=\lambda \kappa .
\end{aligned}
$$

Thus $\kappa=0$. Consequently $\operatorname{grad}\{X(f)\}=0$ if and only if $X(f)=0$. This establishes Assertion (2c).

2.3. The proof of Lemma 2 (3). We have the Bochner identity:

$$
\frac{1}{2} \Delta g(\nabla f, \nabla f)=\left\|\operatorname{Hess}_{f}\right\|^{2}+\rho(\nabla f, \nabla f)+g(\nabla \Delta f, \nabla f) .
$$

By Assertion (1), $\operatorname{Ric}(\nabla f)=0$ and $\|\nabla f\|^{2}-2 \lambda f=$ const. Thus the left-hand side of Equation (6) becomes $\frac{1}{2} \Delta g(\nabla f, \nabla f)=\lambda \Delta f-\frac{1}{2} \Delta \tau$. Taking the trace in Equation (11) shows that $\Delta f=(n+2) \lambda-\tau$ and hence $\frac{1}{2} \Delta g(\nabla f, \nabla f)=\lambda((n+2) \lambda-\tau)$. On the other hand, since $\operatorname{Ric}(\nabla f)=0$ and $\nabla \Delta f=-\nabla \tau=0$, the right-hand side in Bochner formula reduces to $\left\|\operatorname{Hess}_{f}\right\|^{2}$.

2.4. The proof of Lemma 2 (4). If $\|\nabla f\|^{2}=0$, we may apply Assertion (1) to see $2 \lambda f=$ const. Since $\lambda \neq 0, f$ is constant and $(M, g)$ is Einstein. 
2.5. The proof of Lemma 2 (5). If $\lambda=0$, then $\left\|\operatorname{Hess}_{f}\right\|^{2}=0$. By Equation (1), $\mathcal{H}_{f}=-\operatorname{Ric}$ and thus $\operatorname{Ric}(\nabla f)=0$ implies $\mathcal{H}_{f}(\nabla f)=0$. Consequently $\nabla f$ is a geodesic vector field. Next, using the identity $\tau+\|\nabla f\|^{2}-2 \lambda f=$ const, one has that $\|\nabla f\|^{2}$ is constant and therefore $f$ is a solution of the Eikonal equation $\|\nabla f\|^{2}=\mu$.

\section{Non-STEAdy LOCALly homogeneous gRAdient RicCi SOlitons THE PROOF OF TheOREMS 35}

By Lemma 2, isotropic non-steady locally homogeneous gradient Ricci solitons are Einstein. Consequently, we shall concentrate henceforth on the study of nonisotropic non-steady locally homogeneous gradient Ricci solitons. In Section 3.1, we will prove Theorem 3, in Section 3.2 we will establish Theorem 4, and in Section 3.3. we will establish Theorem 5 . We shall use Lemma 2 repeatedly and without further reference in what follows. Throughout Section 3, we shall let $(M, g, f)$ be a locally homogeneous non-steady gradient Ricci soliton.

3.1. The proof of Theorem 3 . Assume that $(M, g)$ is irreducible or, equivalently, that there are no non-trivial parallel distributions on $M$. Consequently any parallel vector field is trivial. Let $X$ be a Killing vector field. Then $\operatorname{grad}\{X(f)\}$ is a parallel vector field and thus $\operatorname{grad}\{X(f)\}=0$ so $X(f)$ is constant and hence $X(f)=0$. Since the underlying Lorentzian structure $(M, g)$ is locally homogeneous, there are $(n+2)$ linearly independent Killing vector fields $X_{1}, \ldots, X_{n+2}$ locally. Consequently $f$ is constant and the metric is Einstein. This establishes Assertion (1) of Theorem 3

We now apply the local splitting result of Assertion (2) in Theorem 1 Let $X$ be a Killing vector field on $(M, g)$. If grad $\{X(f)\}$ is spacelike or timelike, then we may split, at least locally, a one-dimensional factor from $(M, g)$ and decompose locally

$$
(M, g, f)=\left(N \times \mathbb{R}, g_{N} \oplus g_{e}, f_{N}+f_{e}\right) .
$$

If $\operatorname{grad}\{X(f)\}$ is timelike, then $\left(N, g_{N}\right)$ is Riemannian and by Assertion (1) of Theorem 1 rigid which would finish the discussion. Thus we may assume $\left(N, g_{N}\right)$ is Lorentzian so $\operatorname{grad}\{X(f)\}$ is spacelike and the factor $\left(\mathbb{R}, g_{e}\right)$ is positive definite. We proceed inductively to decompose $(M, g, f)=\left(N \times \mathbb{R}^{k}, g_{N} \oplus g_{e}, f_{N}+f_{e}\right)$ (at least locally) so that $\left(N, g_{N}, f_{N}\right)$ is a locally homogeneous Lorentzian Ricci soliton with grad $\{X(f)\}$ null or zero for all Killing vector fields $X$. Now two possibilities may occur. If $N$ is indecomposable, Assertion (3) follows with trivial $N_{1}$. If $N$ is decomposable, then either $N$ is Einstein and Assertion (2) holds (this is the case if $\operatorname{grad}\{X(f)\}=0$ for all Killing vector fields in $N$ ) or $N$ decomposes as $N=N_{0} \times N_{1}$ where $N_{0}$ is Lorentzian and indecomposable (the latter happens if there exists a Killing vector field $X$ so that $\operatorname{grad}\{X(f)\}$ is null). $\left(N_{1}, g_{1}, f_{1}\right)$ is a Riemannian locally homogeneous gradient Ricci soliton which, as a consequence of Theorem 1 is Einstein. This establishes Theorem 3

3.2. The proof of Theorem 4. We establish Assertions (1)-(5) of Theorem 4 seriatim. We suppose $(M, g)$ is not decomposable and is not Einstein.

The proof of Theorem 4 (1). We must show there exists $X$ so $U=\operatorname{grad}\{X(f)\}$ is a parallel null vector field. Let $Z$ be any Killing vector field. Since $(M, g)$ is not decomposable and since $\operatorname{grad}\{Z(f)\}$ is parallel, $\operatorname{grad}\{Z(f)\}$ must be isotropic. If $\operatorname{grad}\{Z(f)\}$ vanishes for all such $Z$, then $f$ is constant and hence $(M, g)$ is Einstein 
which is contrary to our assumption. Thus $U:=\operatorname{grad}\{Z(f)\}$ has the desired properties for some Killing vector field $Z$.

The proof of Theorem 4 (2). We must show that $U$ is unique up to scale, that $U \in \operatorname{ker}\{\operatorname{Ric}\}$, and that $\operatorname{grad}\{U(f)\}=\lambda U$. Suppose that there are two Killing vector fields $Z_{1}$ and $Z_{2}$ on $(M, g)$ so that $\operatorname{grad}\left\{Z_{1}(f)\right\}$ and $\operatorname{grad}\left\{Z_{2}(f)\right\}$ are linearly independent. Since the signature is Lorentzian, $\operatorname{Span}\left\{\operatorname{grad}\left\{Z_{1}(f)\right\}, \operatorname{grad}\left\{Z_{2}(f)\right\}\right\}$ can not be a null distribution. Consequently, there exists a linear combination $Z=a_{1} Z_{1}+a_{2} Z_{2}$ so $\operatorname{grad}\{Z(f)\}$ is either timelike or spacelike. This implies that $(M, g)$ is decomposable which is false. Thus the vector field $U=\operatorname{grad}\{Z(f)\}$ is unique up to scale.

Since $U$ is parallel, it is Killing and hence $\operatorname{grad}\{U(f)\}=\alpha U$ for some $\alpha \in \mathbb{R}$. We must now show that $\operatorname{Ric}(U)=0$. Let $\left\{Z_{1}, Z_{2}, \ldots, Z_{n+2}\right\}$ be a local basis of Killing vector fields. Choose the notation so $Z=Z_{1}$. We then have $\operatorname{grad}\left\{Z_{i}(f)\right\}=\mu_{i} U$ for $i \geq 2$. Since $\operatorname{grad}\left\{Z_{i}(f)\right\}$ is parallel, necessarily $\mu_{i}$ is constant. By replacing $Z_{i}$ by $Z_{i}-\mu_{i} Z_{1}$, we may assume therefore that $\operatorname{grad}\left\{Z_{i}(f)\right\}=0$ for $i \geq 2$. Since $\lambda \neq 0$, Lemma 2 implies $Z_{i}(f)=0$ for $i \geq 2$. We use Equation (11) and Equation (2) to see:

$$
\begin{aligned}
& g(U, \nabla f)=g\left(\operatorname{grad}\left\{Z_{1}(f)\right\}, \nabla f\right)=g\left(\operatorname{grad}\left\{g\left(Z_{1}, \nabla f\right)\right\}, \nabla f\right) \\
& \quad=\nabla f g\left(Z_{1}, \nabla f\right)=g\left(\nabla_{\nabla f} Z_{1}, \nabla f\right)+g\left(Z_{1}, \nabla_{\nabla f} \nabla f\right) \\
& \quad=\operatorname{Hess}_{f}\left(Z_{1}, \nabla f\right)=\lambda g\left(Z_{1}, \nabla f\right)=\lambda Z_{1}(f) \neq 0,
\end{aligned}
$$

where by Equation (3), $g\left(\nabla_{\nabla f} Z_{1}, \nabla f\right)=0$ since $Z_{1}$ is Killing. As $g(U, \nabla f) \neq 0$ and as $U$ is a null vector, $\mathcal{V}:=\operatorname{Span}\{U, \nabla f\}$ has Lorentzian signature. We have that $\operatorname{grad}\{U(f)\} \neq 0$ due to Lemma 2 so $\alpha \neq 0$.

If $X$ is an arbitrary vector field, we study $\mathcal{H}_{f}(U)$ by computing:

$$
\operatorname{Hess}_{f}(X, U)=g\left(U, \nabla_{X} \nabla f\right)=X g(U, \nabla f)=g(X, \operatorname{grad}\{U(f)\})=\alpha g(X, U) .
$$

This shows that $\mathcal{H}_{f}(U)=\alpha U$. Since $\mathcal{H}_{f}(\nabla f)=\lambda \nabla f$, we also have:

$$
\alpha g(\nabla f, U)=\operatorname{Hess}_{f}(\nabla f, U)=\lambda g(\nabla f, U)
$$

so $\alpha=\lambda$. By Equation (11), $\operatorname{Ric}(U)=0$. Since $\nabla_{U} U=0$ and $\nabla_{U} \nabla f=\lambda U, \nabla_{U}$ preserves $\mathcal{V} \subset \operatorname{ker}\{$ Ric $\}$. This proves Assertion (2).

The proof of Theorem 4 (3). We have shown that $\mathcal{V}:=\operatorname{Span}\{U, V\} \subset \operatorname{ker}\{\operatorname{Ric}\}$ is a $U$-parallel Lorentzian distribution. Consequently $\mathcal{V}^{\perp}$ is a Ric invariant distribution with a positive definite signature. Since Ric is self-adjoint, there exists an orthonormal basis $\left\{E_{1}, \ldots, E_{n}\right\}$ of $\mathcal{V}^{\perp}$ so $\operatorname{Ric}\left(E_{i}\right)=\alpha_{i} E_{i}$; the $\alpha_{i}$ are constant since $(M, g)$ is locally homogeneous. This proves in particular that Ric and $\mathcal{H}_{f}=\lambda \mathrm{Id}-\mathrm{Ric}$ are diagonalizable. We now show that $\nabla_{U}$ preserves the eigenspaces in $\mathcal{V}^{\perp}$. For $i \neq j$, since $U$ is parallel $R\left(U, E_{i}, E_{j}, \nabla f\right)=0$. By Lemma 2 (1):

$$
\begin{aligned}
0= & R\left(U, E_{i}, E_{j}, \nabla f\right)=\left(\nabla_{U} \rho\right)\left(E_{i}, E_{j}\right)-\left(\nabla_{E_{i}} \rho\right)\left(U, E_{j}\right) \\
= & U \rho\left(E_{i}, E_{j}\right)-\rho\left(\nabla_{U} E_{i}, E_{j}\right)-\rho\left(E_{i}, \nabla_{U} E_{j}\right) \\
& \quad-E_{i} \rho\left(U, E_{j}\right)+\rho\left(\nabla_{E_{i}} U, E_{j}\right)+\rho\left(U, \nabla_{E_{i}} E_{j}\right) \\
= & -\alpha_{j} g\left(\nabla_{U} E_{i}, E_{j}\right)-\alpha_{i} g\left(E_{i}, \nabla_{U} E_{j}\right) \\
= & \left(\alpha_{i}-\alpha_{j}\right) g\left(\nabla_{U} E_{i}, E_{j}\right) .
\end{aligned}
$$

We conclude that if $E_{i}$ and $E_{j}$ belong to different eigenspaces $\nabla_{U} E_{i}$ is orthogonal to $E_{j}$. Hence, $\nabla_{U}$ commutes with Ric and, as a consequence of the Ricci soliton 
equation (11), it also commutes with $\mathcal{H}_{f}$. Consequently, as desired, $\nabla_{U}$ Ric $=0$ and $\nabla_{U} \mathcal{H}_{f}=0$.

We must show that 0 and $\lambda$ are the only eigenvalues of Ric. Normalize $V$ to be a multiple of $\nabla f$ so $g(V, V)=\epsilon= \pm 1$. Let $S$ be any level set of $f$. The integral curves of $U$ are transversal to $S$ because $g(U, \nabla f) \neq 0$. Use parallel transport along the integral curves of $U$ to extend the local frame $\left\{E_{1}, \ldots, E_{n}\right\}$ from $S$ to a neighborhood of $S$ to define a local frame field $\left\{F_{1}, \ldots, F_{n}\right\}$ for $\mathcal{V}^{\perp}$ such that $\nabla_{U} F_{i}=0$. Since $\nabla_{U}$ Ric $=0$, the vector fields $F_{i}$ are still eigenvectors of the Ricci operator Ric. We shall use this local frame field to see that Ric has only two eigenvalues $\{0, \lambda\}$. First note that

$$
\begin{aligned}
& \left(\nabla_{\nabla f} \rho\right)\left(F_{i}, F_{i}\right)=\nabla f \rho\left(F_{i}, F_{i}\right)-2 \rho\left(\nabla_{\nabla f} F_{i}, F_{i}\right) \\
= & \alpha_{i} \nabla f g\left(F_{i}, F_{i}\right)-2 \alpha_{i} g\left(\nabla_{\nabla f} F_{i}, F_{i}\right)=\alpha_{i}\left(\nabla_{\nabla f} g\right)\left(F_{i}, F_{i}\right)=0 .
\end{aligned}
$$

We use Lemma 2 to compute:

$$
\begin{aligned}
\rho\left(F_{i},\right. & \left.F_{i}\right)=\epsilon R\left(F_{i}, V, F_{i}, V\right) \\
& +\sum_{j \neq i} R\left(F_{i}, F_{j}, F_{i}, V\right) g\left(F_{j}, V\right)+\sum_{j \neq i} R\left(F_{i}, F_{j}, F_{i}, F_{j}\right) \\
= & \frac{\epsilon}{\|\nabla f\|^{2}}\left(\left(\nabla_{F_{i}} \rho\right)\left(\nabla f, F_{i}\right)-\left(\nabla_{\nabla f} \rho\right)\left(F_{i}, F_{i}\right)\right) \\
& +\sum_{j \neq i} R\left(F_{i}, F_{j}, F_{i}, V\right) g\left(F_{j}, V\right)+\sum_{j \neq i} R\left(F_{i}, F_{j}, F_{i}, F_{j}\right) \\
= & \frac{\epsilon}{\|\nabla f\|^{2}}\left(F_{i} \rho\left(\nabla f, F_{i}\right)-\rho\left(\nabla_{F_{i}} \nabla f, F_{i}\right)-\rho\left(\nabla f, \nabla_{F_{i}} F_{i}\right)\right) \\
& +\sum_{j \neq i} R\left(F_{i}, F_{j}, F_{i}, V\right) g\left(F_{j}, V\right)+\sum_{j \neq i} R\left(F_{i}, F_{j}, F_{i}, F_{j}\right) \\
= & -\frac{\epsilon}{\|\nabla f\|^{2}} \rho\left(\mathcal{H}_{f} F_{i}, F_{i}\right) \\
& +\sum_{j \neq i} R\left(F_{i}, F_{j}, F_{i}, V\right) g\left(F_{j}, V\right)+\sum_{j \neq i} R\left(F_{i}, F_{j}, F_{i}, F_{j}\right) .
\end{aligned}
$$

Since we have shown that $\nabla_{U} \rho=0$, we have that $U \rho\left(F_{i}, F_{i}\right)=2 \rho\left(\nabla_{U} F_{i}, F_{i}\right)$ which vanishes. We now differentiate the three summands in the previous expression with respect to $U$ :

$$
\begin{aligned}
& U(-\left.\frac{1}{\|\nabla f\|^{2}} \rho\left(\mathcal{H}_{f} F_{i}, F_{i}\right)\right)=\frac{U g(\nabla f, \nabla f)}{\|\nabla f\|^{4}} \rho\left(\mathcal{H}_{f} F_{i}, F_{i}\right)-\frac{1}{\|\nabla f\|} U \rho\left(\mathcal{H}_{f} F_{i}, F_{i}\right) \\
&= \frac{2 \lambda g(U, \nabla f)}{\|\nabla f\|^{4}} \rho\left(\mathcal{H}_{f} F_{i}, F_{i}\right)-\frac{1}{\|\nabla f\|}\left(\rho\left(\nabla_{U} \mathcal{H}_{f} F_{i}, F_{i}\right)+\rho\left(\nabla_{F_{i}} \nabla f, \nabla_{U} F_{i}\right)\right) \\
&= \frac{2 \lambda g(U, \nabla f)}{\|\nabla f\|^{4}} \rho\left(\mathcal{H}_{f} F_{i}, F_{i}\right)-\frac{1}{\|\nabla f\|}\left(\rho\left(\mathcal{H}_{f}\left(\nabla_{U} F_{i}\right), F_{i}\right)+\rho\left(\nabla_{F_{i}} \nabla f, \nabla_{U} F_{i}\right)\right) \\
&= \frac{2 \lambda g(U, \nabla f)}{\|\nabla f\|^{4}} \alpha_{i}\left(\lambda-\alpha_{i}\right) . \\
& U\left(R\left(F_{i}, F_{j}, F_{i}, \nabla f\right) g\left(F_{j}, \nabla f\right)\right) \\
&=\left\{\left(\nabla_{U} R\right)\left(F_{i}, F_{j}, F_{i}, \nabla f\right)+R\left(\nabla_{U} F_{i}, F_{j}, F_{i}, \nabla f\right)+R\left(F_{i}, \nabla_{U} F_{j}, F_{i}, \nabla f\right)\right. \\
&\left.+R\left(F_{i}, F_{j}, \nabla_{U} F_{i}, \nabla f\right)+R\left(F_{i}, F_{j}, F_{i}, \nabla_{U} \nabla f\right)\right\} g\left(F_{j}, \nabla f\right) \\
&+R\left(F_{i}, F_{j}, F_{i}, \nabla f\right)\left(g\left(\nabla_{U} F_{j}, \nabla f\right)+g\left(F_{j}, \nabla_{U} \nabla f\right)\right) \\
&=\left\{-\left(\nabla_{F_{i}} R\right)\left(F_{j}, U, F_{i}, \nabla f\right)-\left(\nabla_{F_{j}} R\right)\left(U, F_{i}, F_{i}, \nabla f\right)\right. \\
&+R\left(\nabla_{U} F_{i}, F_{j}, F_{i}, \nabla f\right)+R\left(F_{i}, \nabla_{U} F_{j}, F_{i}, \nabla f\right)+R\left(F_{i}, F_{j}, \nabla_{U} F_{i}, \nabla f\right) \\
&\left.+R\left(F_{i}, F_{j}, F_{i}, \lambda U\right)\right\} g\left(F_{j}, \nabla f\right) \\
&+R\left(F_{i}, F_{j}, F_{i}, \nabla f\right)\left(g\left(\nabla_{U} F_{j}, \nabla f\right)+\lambda g\left(F_{j}, U\right)\right) \\
&=\left\{R\left(\nabla_{U} F_{i}, F_{j}, F_{i}, \nabla f\right)+R\left(F_{i}, \nabla_{U} F_{j}, F_{i}, \nabla f\right)\right. \\
&\left.+R\left(F_{i}, F_{j}, \nabla_{U} F_{i}, \nabla f\right)\right\} g\left(F_{j}, \nabla f\right)+R\left(F_{i}, F_{j}, F_{i}, \nabla f\right) g\left(\nabla_{U} F_{j}, \nabla f\right) \\
&= 0 .
\end{aligned}
$$


Consequently along the slice $S$ we have:

$$
\begin{aligned}
U( & \left.R\left(F_{i}, F_{j}, F_{i}, V\right) g\left(F_{j}, V\right)\right)=U\|\nabla f\|^{-2} R\left(F_{i}, F_{j}, F_{i}, \nabla f\right) g\left(F_{j}, \nabla f\right) \\
\quad & +\|\nabla f\|^{-2} U\left(R\left(F_{i}, F_{j}, F_{i}, \nabla f\right) g\left(F_{j}, \nabla f\right)\right)=0 \\
U R & \left(F_{i}, F_{j}, F_{i}, F_{j}\right)=\left(\nabla_{U} R\right)\left(F_{i}, F_{j}, F_{i}, F_{j}\right)+2 R\left(\nabla_{U} F_{i}, F_{j}, F_{i}, F_{j}\right) \\
\quad & +2 R\left(F_{i}, \nabla_{U} F_{j}, F_{i}, F_{j}\right) \\
= & -\left(\nabla_{F_{i}} R\right)\left(F_{j}, U, F_{i}, F_{j}\right)-\left(\nabla_{F_{j}} R\right)\left(U, F_{i}, F_{i}, F_{j}\right) \\
& +2 R\left(\nabla_{U} F_{i}, F_{j}, F_{i}, F_{j}\right)+2 R\left(F_{i}, \nabla_{U} F_{j}, F_{i}, F_{j}\right) \\
= & 2 R\left(\nabla_{U} F_{i}, F_{j}, F_{i}, F_{j}\right)+2 R\left(F_{i}, \nabla_{U} F_{j}, F_{i}, F_{j}\right) \\
= & 0 .
\end{aligned}
$$

Hence, the following equation holds:

$$
0=2 \lambda g(U, \nabla f)\|\nabla f\|^{-4} \alpha_{i}\left(\lambda-\alpha_{i}\right) .
$$

Since $\lambda$ and $g(U, \nabla f)$ are different from 0 , either $\alpha_{i}=0$ or $\alpha_{i}=\lambda$ for $i=1, \ldots, n$. Since the level set $S$ of $f$ which was chosen was arbitrary, this is true on all of $M$. By Equation (11) we have $\mathcal{H}_{f}+\mathrm{Ric}=\lambda \mathrm{Id}$. The remaining conclusions of Assertion (3) are now immediate from the discussion above.

3.2.1. The proof of Theorem 4 (4). Recall that $(M, g)$ has a harmonic Weyl tensor if its Schouten tensor $S=\rho-\frac{\tau}{2(n+1)} g$ is Codazzi, i.e., $\nabla_{X} S_{Y Z}=\nabla_{Y} S_{X Z}$ (see 2] $)$. If the Weyl tensor is harmonic then $\left(\nabla_{X} \rho\right)(Y, Z)-\left(\nabla_{Y} \rho\right)(X, Z)=0$ since the scalar curvature is constant. Choose $E_{1}, E_{2} \in \operatorname{Image}\left\{\mathcal{H}_{f}\right\}$ and $F \in \operatorname{Image}\{$ Ric $\}$. We use Assertion (3) to compute

$$
0=\left(\nabla_{E_{1}} \rho\right)\left(F, E_{2}\right)-\left(\nabla_{F} \rho\right)\left(E_{1}, E_{2}\right)=\rho\left(F, \nabla_{E_{1}} E_{2}\right)=\lambda g\left(F, \nabla_{E_{1}} E_{2}\right) .
$$

Choose $E \in \operatorname{Image}\left\{\mathcal{H}_{f}\right\}$ and $F_{1}, F_{2} \in$ Image $\{$ Ric $\}$. We show the two eigenspaces are parallel and that the soliton is rigid by computing:

$$
\begin{aligned}
0 & =\left(\nabla_{F_{1}} \rho\right)\left(E, F_{2}\right)-\left(\nabla_{E} \rho\right)\left(F_{1}, F_{2}\right) \\
& =\rho\left(\nabla_{F_{1}} E, F_{2}\right)-E \rho\left(F_{1}, F_{2}\right)+\rho\left(\nabla_{E} F_{1}, F_{2}\right)+\rho\left(F_{1}, \nabla_{E} F_{2}\right) \\
& =\lambda g\left(\nabla_{F_{1}} E, F_{2}\right)-\lambda E g\left(F_{1}, F_{2}\right)+\lambda g\left(\nabla_{E} F_{1}, F_{2}\right)+\lambda g\left(F_{1}, \nabla_{E} F_{2}\right) \\
& =\lambda g\left(\nabla_{F_{1}} E, F_{2}\right) .
\end{aligned}
$$

The proof of Theorem 4 (5). We apply Theorem 3 , If $\operatorname{dim}(\operatorname{ker}\{\operatorname{Ric}\})=2$, then $\mathcal{V}=\operatorname{ker}\{$ Ric $\}$. Since $U$ is parallel, we have that $\mathcal{H}_{f}(X)=\nabla_{X} \nabla f=\lambda X$ if $X \in \mathcal{V}$ and that $\mathcal{H}_{f}(X)=\nabla_{X} \nabla f=0$ if $X \in \operatorname{ker}\left\{\mathcal{H}_{f}\right\}=$ Image $\{$ Ric $\}$. Consequently, the distribution $\mathcal{V}$ is parallel. Since the metric is not degenerate on $\mathcal{V}$, this implies that the manifold locally decomposes as a product $B \times F$ so that $B$ is Ricci flat and hence flat. On the other hand $F$ is Einstein satisfying $\rho^{F}=\lambda g^{F}$. Therefore the soliton is rigid. This completes the proof of Theorem 4 .

3.3. The proof of Theorem 5. If $\operatorname{dim}(M)=3$ the result follows from the discussion above since $\operatorname{dim}(\operatorname{ker}\{\operatorname{Ric}\})=2$. Assume $\operatorname{dim}(M)=4$ henceforth. Using the previous discussion, we need only examine the case $\operatorname{dim}(\operatorname{ker}\{\operatorname{Ric}\})=3$. We are going to use Theorem 4 to show that Image $\{\mathrm{Ric}\}$ is a non-null parallel distribution. We consider the adapted basis $\{U, \nabla f, E, F\}$ where $\{U, \nabla f, E\}$ is a basis of $\operatorname{ker}\{$ Ric $\}$ and $F \cdot \mathbb{R}=$ Image $\{$ Ric $\}$. We show that the Weyl tensor is harmonic and $(M, g, f)$ is rigid by examining the components of the curvature tensor which have $\nabla f$ as an argument: 


$$
\begin{aligned}
& R(E, \nabla f, E, \nabla f)=\left(\nabla_{E} \rho\right)(\nabla f, E)-\left(\nabla_{\nabla f} \rho\right)(E, E)=0, \\
& R(F, \nabla f, F, \nabla f)=\left(\nabla_{F} \rho\right)(\nabla f, F)-\left(\nabla_{\nabla f} \rho\right)(F, F)=0, \\
& R(F, \nabla f, E, \nabla f)=\rho(F, E)\|\nabla f\|^{2}=0, \\
& R(F, E, F, \nabla f)=\rho(\nabla f, E)=0, \quad R(E, F, E, \nabla f)=\rho(\nabla f, F)=0 .
\end{aligned}
$$

\section{Steady locally homogeneous Lorentzian gradient Ricci solitons The proof of Theorems 89}

Again, we shall use Lemma 2 throughout the section without further citation. Let $(M, g, f)$ be a steady locally homogeneous Lorentzian gradient Ricci soliton. Then $\left\|\operatorname{Hess}_{f}\right\|^{2}=0$ and $\|\nabla f\|^{2}=\mu$ is constant. In what follows we will consider the possibilities $\mu<0$ and $\mu=0$ separately.

4.1. The proof of Theorem 8, Assume that $\mu<0$. As $\mathcal{H}_{f}(\nabla f)=0$, we may restrict $\mathcal{H}_{f}$ to $\nabla f^{\perp}$. As $\nabla f^{\perp}$ inherits a positive definite metric and since $\left\|\operatorname{Hess}_{f}\right\|^{2}=0, \mathcal{H}_{f}=0$. This shows that $\nabla f$ is a parallel vector field, and thus $(M, g)$ is locally a product $\left(\mathbb{R} \times N,-d t^{2}+g_{N}\right)$, where $\left(N, g_{N}\right)$ is a locally homogeneous Riemannian manifold (see, for example, [18). Additionally, $\left(N, g_{N}\right)$ is a steady gradient Ricci soliton, and therefore Ricci flat. Following [26, locally homogeneous Ricci flat Riemannian manifolds are locally isometric to Euclidean space. This completes the proof of Theorem 8 .

4.2. The proof of Theorem 9 (1). Assume that $\|\nabla f\|^{2}=0$ so $\nabla f$ is a null vector. Choose an orthonormal basis $\left\{E_{1}, \ldots, E_{n+2}\right\}$ for the tangent space at a point so $E_{1}$ is timelike, so $\left\{E_{2}, \ldots, E_{n+2}\right\}$ are spacelike, and so $\nabla f=c\left(E_{1}+E_{2}\right)$ for some $c \neq 0$. We further normalize the basis so $\mathcal{H}_{f} E_{1} \in \operatorname{Span}\left\{E_{1}, E_{2}, E_{3}\right\}$. Let $\mathcal{H}_{f} E_{i}=\mathcal{H}_{i}^{j} E_{j}$. Since $E_{1}+E_{2} \in \operatorname{ker}\left\{\mathcal{H}_{f}\right\}, \mathcal{H}_{1}^{i}+\mathcal{H}_{2}^{i}=0$ for all $i$. Furthermore, $\mathcal{H}_{1}^{i}=\mathcal{H}_{2}^{i}=0$ for $i \geq 4$ since $\mathcal{H}_{f} E_{1} \in \operatorname{Span}\left\{E_{1}, E_{2}, E_{3}\right\}$. Finally, since $\mathcal{H}_{f}$ is self-adjoint, $\mathcal{H}_{1}^{i}=-\mathcal{H}_{i}^{1}$ for $2 \leq i$ and $\mathcal{H}_{i}^{j}=\mathcal{H}_{j}^{i}$ for $2 \leq i, j$. We summarize these relations:

$$
\begin{array}{ll}
\mathcal{H}_{1}^{i}=-\mathcal{H}_{i}^{1} \text { for } i \geq 2, & \mathcal{H}_{i}^{j}=\mathcal{H}_{j}^{i} \text { for } 2 \leq i, j, \\
\mathcal{H}_{1}^{i}=\mathcal{H}_{2}^{i}=0 \text { for } i \geq 4, & \mathcal{H}_{1}^{i}+\mathcal{H}_{2}^{i}=0 \text { for all } i .
\end{array}
$$

Since $\mathcal{H}_{f}=\mathcal{H}_{i}^{j} E^{i} \otimes E_{j}$ and $\left\|\operatorname{Hess}_{f}\right\|^{2}=\lambda((n+2) \lambda-\tau)=0$, we have

$$
0=\left\|\operatorname{Hess}_{f}\right\|^{2}=\left\|\mathcal{H}_{f}\right\|^{2}=\left(\mathcal{H}_{1}^{1}\right)^{2}-2 \sum_{i \geq 2}\left(\mathcal{H}_{i}^{1}\right)^{2}+\sum_{2 \leq j, k}\left(\mathcal{H}_{j}^{k}\right)^{2} .
$$

The relations of Equation (8) then permit us to rewrite Equation (9) in the form:

$$
0=\sum_{3 \leq j, k}\left(\mathcal{H}_{j}^{k}\right)^{2} .
$$

This implies $\mathcal{H}_{j}^{k}=0$ for $3 \leq j, k$ and thus by Equation (8), $\mathcal{H}_{f} E_{i}=0$ for $i \geq 4$. Thus the relevant portion of the matrix $\mathcal{H}$ becomes:

$$
\mathcal{H}=\left(\begin{array}{lll}
\mathcal{H}_{1}^{1} & \mathcal{H}_{2}^{1} & \mathcal{H}_{3}^{1} \\
\mathcal{H}_{1}^{2} & \mathcal{H}_{2}^{2} & \mathcal{H}_{3}^{2} \\
\mathcal{H}_{1}^{3} & \mathcal{H}_{2}^{3} & \mathcal{H}_{3}^{3}
\end{array}\right)=\left(\begin{array}{rrr}
\mathcal{H}_{1}^{1} & -\mathcal{H}_{1}^{1} & \mathcal{H}_{3}^{1} \\
\mathcal{H}_{1}^{1} & -\mathcal{H}_{1}^{1} & \mathcal{H}_{3}^{1} \\
-\mathcal{H}_{3}^{1} & \mathcal{H}_{3}^{1} & 0
\end{array}\right)
$$


We compute

$$
\mathcal{H}^{2}=\left(\mathcal{H}_{1}^{3}\right)^{2}\left(\begin{array}{rrr}
-1 & 1 & 0 \\
-1 & 1 & 0 \\
0 & 0 & 0
\end{array}\right) \text { and } \mathcal{H}^{3}=0 .
$$

This shows that $\mathcal{H}$ is either 2 or 3 -step nilpotent which proves Assertion (1).

4.3. The proof of Theorem 9 (2). Let $\mathcal{H}_{f}$ be 2 -step nilpotent. The analysis above shows $\nabla f \in \operatorname{Image}\left\{\mathcal{H}_{f}\right\}$. Since $\mathcal{H}_{f}$ has rank 1 , Image $\left\{\mathcal{H}_{f}\right\}=\nabla f \cdot \mathbb{R}$. We use the Fredholm alternative and the fact that $\mathcal{H}_{f}$ is self-adjoint to establish Assertion (2a) using the following equivalencies:

$$
\begin{aligned}
& \mathcal{H}_{f} Z=0 \quad \Leftrightarrow \quad g\left(\mathcal{H}_{f} Z, Y\right)=0 \forall Y \\
& \Leftrightarrow g\left(Z, \mathcal{H}_{f} Y\right)=0 \forall Y \Leftrightarrow Z \perp \text { Range }\left\{\mathcal{H}_{f}\right\} \\
& \Leftrightarrow Z \perp \nabla f \text {. }
\end{aligned}
$$

Choose a vector field $U$ so $g(U, \nabla f)=1$. Since Range $\left\{\mathcal{H}_{f}\right\}=\nabla f$ and since $g(U, \nabla f)=1$, the fact that $\nabla f$ is recurrent follows from the equation:

$$
\nabla_{X}(\nabla f)=\mathcal{H}_{f}(X)=\theta(X) \cdot \nabla f \text { where } \theta(X)=g\left(U, \mathcal{H}_{f}(X)\right) .
$$

Let $X$ and $Y$ be smooth vector fields in $\nabla f^{\perp}$. We show that $[X, Y]$ belongs to $\nabla f^{\perp}$ and thus $\nabla f^{\perp}$ is an integrable distribution by computing:

$$
\begin{aligned}
& g([X, Y], \nabla f)=g\left(\nabla_{X} Y-\nabla_{Y} X, \nabla f\right) \\
= & X g(Y, \nabla f)-g\left(Y, \nabla_{X} \nabla f\right)-Y g(X, \nabla f)+g\left(X, \nabla_{Y} \nabla f\right) \\
= & X\{0\}-\operatorname{Hess}_{f}(Y, X)-Y\{0\}+\operatorname{Hess}_{f}(X, Y)=0 .
\end{aligned}
$$

Let $\gamma(t)$ be a geodesic with $\dot{\gamma}(0) \perp \nabla f$. We compute

$$
\partial_{t} g(\dot{\gamma}, \nabla f)=g(\ddot{\gamma}, \nabla f)+g\left(\dot{\gamma}, \nabla_{\partial_{t}} \nabla f\right)=\theta\left(\partial_{t}\right) g(\dot{\gamma}, \nabla f) .
$$

Since $g(\dot{\gamma}, \nabla f)(0)=0$, the fundamental theorem of ODE's implies $g(\dot{\gamma}, \nabla f)$ vanishes identically and thus $\dot{\gamma} \in \nabla f^{\perp}$. Since $g(\dot{\gamma}, \nabla f)=\partial_{t} f$, the geodesic lies entirely in the level set of $f$. Assertion (2b) follows.

We proceed by induction on the dimension to establish Assertion (2c). Fix a point $P \in M$. Let $\mathcal{V}:=\operatorname{Span}\{U, \nabla f\}$. The metric on $\mathcal{V}$ is non-degenerate and contains a null vector; consequently $\mathcal{V}$ has Lorentzian signature. We can choose complementary Killing vector fields $\left\{F_{1}, \ldots, F_{n}\right\}$ so $\left\{U, \nabla f, F_{1}, \ldots, F_{n}\right\}$ is a local frame field near $P$ and so that

$$
\left.g\left(U, F_{i}\right)\right|_{P}=\left.g\left(\nabla f, F_{i}\right)\right|_{P}=0 .
$$

Consequently $\operatorname{Span}\left\{F_{1}, \ldots, F_{n}\right\}$ is spacelike near $P$. Let $\xi_{i}:=\operatorname{grad}\left\{F_{i}(f)\right\}$; these are parallel vector fields by Lemma 2 Let $\mathcal{W}:=\operatorname{Span}\left\{\xi_{1}, \ldots, \xi_{n}\right\}$. Since the $\xi_{i}$ are parallel, $r(x):=\operatorname{Rank}\{\mathcal{W}(x)\}$ is locally constant. Suppose $r>0$. By reordering the collection $\left\{F_{1}, \ldots, F_{n}\right\}$ if necessary, we may assume that $\left\{\xi_{1}, \ldots, \xi_{r}\right\}$ is a local frame field for $\mathcal{W}$. Let $\epsilon_{i j}:=g\left(\xi_{i}, \xi_{j}\right)$ describe the induced metric on $\mathcal{W}$. Again we use the fact that the $\xi_{i}$ are parallel; this implies that the $\epsilon_{i j}$ are constant. We can diagonalize $\epsilon$ or equivalently renormalize the choice of the Killing vector fields $F_{i}$ to assume that $\epsilon$ is in fact diagonal. If $\operatorname{det}(\epsilon)=0$, then $\xi_{i}$ is a parallel null vector field for some $i$ and Assertion (2-c-i) holds. Thus we may assume that the inner-product restricted to $\mathcal{W}$ is non-degenerate. We may use Theorem 1 to decompose, at least locally, $M=N^{2+n-r} \times \mathbb{R}_{\nu}^{r}$. If the metric on $N$ is Riemannian, we may apply Theorem 1 to see that the soliton is trivial. Thus $N$ is Lorentzian. If $\operatorname{dim}(N)=2$, then Theorem $\left[7\right.$ shows $N$ is flat and $\mathcal{H}_{f}=0$ which is false. This $\operatorname{shows} \operatorname{dim}(N) \geq 3$ 
and we may use our induction hypothesis on $N$. Thus we may assume without loss of generality that $r=0$ so $\mathcal{W}=\{0\}$ and assume henceforth that:

$$
\operatorname{grad}\left\{F_{i}(f)\right\}=0 \text { for all } i \text {. }
$$

By Equation (12), $\kappa_{i}:=F_{i}(f)$ is constant for all $i$. By Equation (11),

$$
\kappa_{i}=\left.F_{i}(f)\right|_{P}=\left.g\left(F_{i}, \nabla f\right)\right|_{P}=0 .
$$

Consequently $g\left(F_{i}, \nabla f\right)$ vanishes identically and we have

$$
F_{i} \in \operatorname{ker}\left\{\mathcal{H}_{f}\right\}=\operatorname{ker}\{\operatorname{Ric}\}=\nabla f^{\perp} .
$$

We may use Equation (10) and Equation (13) to see

$$
\begin{aligned}
& \nabla_{\nabla f} \nabla f=\mathcal{H}_{f}(\nabla f)=0, \quad \nabla_{F_{i}} \nabla f=\mathcal{H}_{f}\left(F_{i}\right)=0 \text { for all i, } \\
& \nabla_{U} \nabla f=\mathcal{H}_{f}(U)=\Xi \nabla f \text { where } \Xi:=g\left(\mathcal{H}_{f}(U), U\right)=-\rho(U, U) .
\end{aligned}
$$

We use Equation (14) to see:

$$
\nabla_{Y} \nabla f=0 \text { if } Y \perp \nabla f .
$$

Thus the only covariant derivative at issue is $\nabla_{U} \nabla f$. We shall let $\Psi:=\psi \cdot \nabla f$. This is a null vector field. By Equation (15), $\Psi$ will be parallel if and only if $\psi$ satisfies the equations:

$$
Y(\psi)=0 \text { if } Y \perp \nabla f \text { and } U(\psi)+\psi \Xi=0 .
$$

Since $F_{i}$ is a Killing vector field, $\nabla_{F_{i}} \rho=0$. Since $F_{i} \in \operatorname{ker}\{\operatorname{Ric}\}, \rho\left(F_{i}, \cdot\right)$ vanishes identically. Consequently, Lemma 2 yields

$$
\begin{aligned}
& R\left(F_{i}, U, F_{j}, \nabla f\right)=\left(\nabla_{F_{i}} \rho\right)\left(U, F_{j}\right)-\left(\nabla_{U} \rho\right)\left(F_{i}, F_{j}\right) \\
& \quad=-U \rho\left(F_{i}, F_{i}\right)+\rho\left(\nabla_{U} F_{i}, F_{j}\right)+\rho\left(\nabla_{U} F_{j}, F_{i}\right)=0 .
\end{aligned}
$$

Let $g_{i j}=g\left(F_{i}, F_{j}\right)$. Since $U \in \operatorname{ker}\{\operatorname{Ric}\}$, since $\{U, \nabla f\}$ span a hyperbolic pair, Equation (17) implies:

$$
\begin{aligned}
0 & =\left.\rho(U, \nabla f)\right|_{P}=\left.R(U, \nabla f, \nabla f, U)\right|_{P}+\left.\sum_{i, j=1}^{n} g^{i j} R\left(U, F_{i}, \nabla f, F_{j}\right)\right|_{P} \\
& =\left.R(U, \nabla f, \nabla f, U)\right|_{P} .
\end{aligned}
$$

Since $P$ was arbitrary and the only condition on $U$ was that $g(U, \nabla f)=1$, this holds for arbitrary $P$ and we have

$$
0=R(U, \nabla f, \nabla f, U) \text { if } g(U, \nabla f)=1 .
$$

Also, in general, if $X$ is a Killing vector field, then for arbitrary vector fields, we have (see, for example, 20, 22]) that:

$$
R(X, Y) Z=-\nabla_{Y} \nabla_{Z} X+\nabla_{\nabla_{Y} Z} X .
$$

Let $\Xi$ be as defined in Equation(14). We use Equation (13) to see:

$$
g\left(\nabla_{U} F_{i}, \nabla f\right)=U g\left(F_{i}, \nabla f\right)-g\left(F_{i}, \nabla_{U} \nabla f\right)=-g\left(F_{i}, \Xi \nabla f\right)=0 .
$$


Since the $F_{i}$ are Killing vector fields, since $g\left(F_{i}, \nabla f\right)=0$, and since $\nabla f$ is recurrent,

$$
\begin{aligned}
& R\left(F_{i}, U, U, \nabla f\right)=-g\left(\nabla_{U} \nabla_{U} F_{i}, \nabla f\right)+g\left(\nabla_{\nabla_{U} U} F_{i}, \nabla f\right) \\
= & -U g\left(\nabla_{U} F_{i}, \nabla f\right)+g\left(\nabla_{U} F_{i}, \nabla_{U} \nabla f\right) \\
& \quad+\left(\nabla_{U} U\right) g\left(F_{i}, \nabla f\right)-g\left(F_{i}, \nabla_{\nabla_{U} U}\{\nabla f\}\right) \\
= & -U\left\{U g\left(F_{i}, \nabla f\right)-g\left(F_{i}, \nabla_{U} \nabla f\right)\right\}+g\left(\nabla_{U} F_{i}, \Xi \nabla f\right) \\
= & U g\left(F_{i}, \Xi \nabla f\right)+\Xi g\left(\nabla_{U} F_{i}, \nabla f\right)=0 .
\end{aligned}
$$

By Lemma 2, if $\{X, Y, Z\}$ are vector fields on a gradient Ricci soliton, then

$$
R(X, Y, Z, \nabla f)=\left(\nabla_{X} \rho\right)(Y, Z)-\left(\nabla_{Y} \rho\right)(X, Z) .
$$

Consequently, we have that

$$
\begin{aligned}
& 0=R(U, \nabla f, U, \nabla f)=\left(\nabla_{U} \rho\right)(\nabla f, U)-\left(\nabla_{\nabla f} \rho\right)(U, U) \\
& 0=R\left(F_{i}, U, U, \nabla f\right)=\left(\nabla_{F_{i}} \rho\right)(U, U)-\left(\nabla_{U} \rho\right)\left(F_{i}, U\right) .
\end{aligned}
$$

By Equation (14), $\Xi=-\rho(U, U)$. Thus we may compute:

$$
\begin{aligned}
&-\nabla f(\Xi)=\nabla f \rho(U, U)=\left(\nabla_{\nabla f} \rho\right)(U, U)+2 \rho\left(\nabla_{\nabla f} U, U\right) \\
&=\left(\nabla_{U} \rho\right)(\nabla f, U)-2 g\left(\nabla_{\nabla f} U, \Xi \nabla f\right) \\
&= U \rho(\nabla f, U)-\rho\left(\nabla_{U} \nabla f, U\right)-\rho\left(\nabla f, \nabla_{U} U\right) \\
&-2 \Xi\left(\nabla f g(U, \nabla f)-g\left(U, \nabla_{\nabla f} \nabla f\right)\right)=0, \text { and } \\
&-F_{i}(\Xi)=F_{i} \rho(U, U)=\left(\nabla_{F_{i}} \rho\right)(U, U)+2 \rho\left(\nabla_{F_{i}} U, U\right) \\
&=\left(\nabla_{U} \rho\right)\left(F_{i}, U\right)-2 g\left(\nabla_{F_{i}} U, \Xi \nabla f\right) \\
&= U \rho\left(F_{i}, U\right)-\rho\left(\nabla_{U} F_{i}, U\right)-\rho\left(F_{i}, \nabla_{U} U\right) \\
&-2 \Xi\left(F_{i} g(U, \nabla f)-g\left(U, \nabla_{F_{i}} \nabla f\right)\right) \\
&= g\left(\nabla_{U} F_{i}, \Xi \nabla f\right)=\Xi U g\left(F_{i}, \nabla f\right)-\Xi g\left(F_{i}, \Xi \nabla f\right)=0 .
\end{aligned}
$$

This shows that $X(\Xi)=0$ if $X \in \nabla f^{\perp}$. Since the distribution $\nabla f^{\perp}$ is integrable, the Frobenius theorem means we can introduce local coordinates $\left(u, x^{2}, \ldots, x^{n+2}\right)$ so that $U=\partial_{u}$ and $\nabla f^{\perp}=\operatorname{Span}\left\{\partial_{x_{2}}, \ldots, \partial_{x_{n+2}}\right\}$. Thus Equation (16) becomes an ordinary differential equation which can be solved. This completes the proof of Theorem 9

Example 17. We follow the discussion in 11. A Cahen-Wallach space has the metric given locally by Equation (4):

$$
g=2 d t d y+\left(\sum_{i=1}^{n} \kappa_{i} x_{i}^{2}\right) d y^{2}+\sum_{i=1}^{n} d x_{i}^{2} \text { for } 0 \neq \kappa_{i} \in \mathbb{R} .
$$

The Levi-Civita connection is determined by the non-zero Christoffel symbols:

$$
\nabla_{\partial_{y}} \partial_{y}=-\sum_{i} \kappa_{i} x_{i} \partial_{x_{i}} \text { and } \nabla_{\partial_{y}} \partial_{x_{i}}=\nabla_{\partial_{x_{i}}} \partial_{y}=\kappa_{i} x_{i} \partial_{v}
$$

Thus the only non-zero entries in the curvature tensor are given by:

$$
R\left(\partial_{y}, \partial_{x_{i}}, \partial_{y}, \partial_{x_{i}}\right)=-\kappa_{i}
$$

and thus (possibly) non-zero entries in the Ricci tensor are

$$
\rho\left(\partial_{y}, \partial_{y}\right)=-\kappa \text { where } \kappa:=\kappa_{1}+\ldots+\kappa_{n} .
$$

Assuming that $\kappa \neq 0$, we then have $\operatorname{Ric}\left(\partial_{y}\right)=-\kappa \partial_{t}$ and $\operatorname{Ric}\left(\partial_{t}\right)=0$. Thus the Ricci tensor is two step nilpotent. The $f$ defines a gradient Ricci soliton if and only 
if $f\left(t, y, x_{1}, \ldots, x_{n}\right)=f(y)$ where $f(y)=a_{0}+a_{1} y+\frac{1}{4} \kappa y^{2} ; \lambda=0$ in this instance. Note that $d f=\left(a_{1}+\frac{1}{2} \kappa y\right) d y$ and hence $\nabla f=\left(a_{1}+\frac{1}{2} \kappa y\right) \partial_{t}$ is a null parallel vector field.

\section{SyMmetriC GRADient RiCCI SOLitons THE PROOF OF THEOREM 12}

Let $(M, g)$ be a locally symmetric Lorentzian manifold. If $(M, g, f)$ is a nonsteady gradient Ricci soliton, then by Theorem 3. $M$ splits, at least locally, as a product $M=N_{0} \times N_{1} \times \mathbb{R}^{k}$, where $\left(N_{0}, g_{0}\right)$ is indecomposable but reducible and $\left(N_{1}, g_{1}\right)$ is Einstein. If $N_{0}$ does not appear in the decomposition, then the soliton is rigid. Otherwise, $\left(N_{0}, g_{0}\right)$ is an indecomposable but not irreducible Lorentzian symmetric space, hence a Cahen-Wallach symmetric space [7] (see also [3]). Theorem 11 rules out this latter possibility since if $\left(N, g_{N}, f_{N}\right)$ is a Cahen-Wallach gradient Ricci soliton, then it is steady.

Next suppose that $(M, g, f)$ is a locally symmetric Lorentzian steady gradient Ricci soliton. We can use the de Rham-Wu decomposition of the manifold to split $(M, g)$ locally as a product $M=N \times M_{1} \times \cdots \times M_{l} \times \mathbb{R}_{\nu}^{k}$, where $\left(N, g_{N}\right)$ is a CahenWallach symmetric space, where the $M_{i}$ are irreducible symmetric spaces, and where $\mathbb{R}_{\nu}^{k}$ is either Euclidean or Minkowskian space. Since irreducible symmetric spaces are Einstein, the induced soliton is either trivial or the scalar curvature vanishes, which implies that $M_{i}$ is Ricci flat. If $M_{i}$ is Riemannian, then it is flat since Ricci flat locally symmetric spaces are flat in the Riemannian setting [2, 19]. Moreover, if $M_{i}$ is Lorentzian, then it is flat since irreducible Lorentzian locally symmetric spaces are of constant sectional curvature [8]. Hence, if the gradient Ricci soliton is steady, then the decomposition above reduces to $M=N \times \mathbb{R}^{k}$, where $\left(N, g_{N}\right)$ is a Cahen-Wallach symmetric space. Theorem 12 now follows.

\section{ThreE-DimensionAl LOCALLY HOMOgEnEOUS GRADIENT RiCCI SOLITONS}

6.1. The proof of Theorem 13. Let $(M, g)$ be a 3-dimensional Lorentzian strict Walker metric. There exist local coordinates so the metric is given by Equation (5):

$$
g=2 d t d y+d x^{2}+\phi(x, y) d y^{2} .
$$

Let $f(t, x, y)$ be a smooth real valued function. To simplify the notation, set $f_{t}=\frac{\partial f}{\partial t}, f_{t x}=\frac{\partial^{2} f}{\partial t \partial x}$, and so forth. One computes easily that the soliton equation $\operatorname{Hess}_{f}+\rho=\lambda g$ is equivalent to the following relations:

$$
\begin{array}{ll}
0=f_{t t}=f_{t x}, & 0=f_{x x}-\lambda=f_{t y}-\lambda, \\
0=2 f_{x y}-\phi_{x} f_{t}, & 0=2 \lambda \phi+\phi_{x x}-2 f_{y y}-\phi_{x} f_{x}+\phi_{y} f_{t} .
\end{array}
$$

We use the first identities in Equation (19) to see:

$$
f(t, x, y)=t(\lambda y+\kappa)+\frac{1}{2} \lambda x^{2}+\alpha(y) x+\gamma(y) \text { for } \kappa \in \mathbb{R} .
$$

Hence, the equations of Equation (19) simplify to become:

$$
\begin{aligned}
& 0=2 \alpha^{\prime}(y)-(\lambda y+\kappa) \phi_{x}, \\
& 0=2 \lambda \phi-2 \gamma^{\prime \prime}(y)-2 x \alpha^{\prime \prime}(y)+(\lambda y+\kappa) \phi_{y}-(\lambda x+\alpha(y)) \phi_{x}+\phi_{x x} .
\end{aligned}
$$

We differentiate Equation (20) with respect to $x$ to conclude:

$$
0=(\lambda y+\kappa) \phi_{x x} .
$$


Since the Ricci operator is given by:

$$
\text { Ric }=\left(\begin{array}{ccc}
0 & 0 & -\frac{1}{2} \phi_{x x} \\
0 & 0 & 0 \\
0 & 0 & 0
\end{array}\right),
$$

the metric is flat if and only if $\phi_{x x}=0$. Since we assume that the Walker metric is not-flat, we may use Equation (22) to see that $\lambda=\kappa=0$ and conclude that the gradient Ricci soliton is steady. Consequently Equations (20) and (22) imply that $f(t, x, y)=\alpha x+\gamma(y)$ so Equation (21) becomes:

$$
2 \gamma^{\prime \prime}(y)+\alpha \phi_{x}-\phi_{x x}=0 .
$$

We take the derivative with respect to $x$ to see $\alpha \phi_{x x}=\phi_{x x x}$. We examine the two cases seriatim.

Case I: Suppose that $\alpha \neq 0$. We then have:

$$
\phi(x, y)=\frac{1}{\alpha^{2}} a(y) e^{\alpha x}+x b(y)+c(y)
$$

for some arbitrary functions $a(y) \neq 0, b(y)$ and $c(y)$. Moreover the potential function of the soliton is given by $f(t, x, y)=\alpha x+\gamma(y)$, where $\gamma^{\prime \prime}(y)=-\frac{1}{2} \alpha b(y)$. In this case $\nabla f=\gamma^{\prime}(y) \partial_{t}+\alpha \partial_{x}$ is spacelike. This gives rise to the first possibility in Theorem 13

Case II: Suppose that $\alpha=0$. We then have:

$$
\phi(x, y)=x^{2} a(y)+x b(y)+c(y)
$$

for some arbitrary functions $a(y) \neq 0, b(y)$ and $c(y)$. Moreover the potential function of the soliton is given by $f(t, x, y)=\gamma(y)$, where $\gamma^{\prime \prime}(y)=\frac{1}{4} a(y)$. In this case $\nabla f=\gamma^{\prime}(y) \partial_{t}$ is a null and recurrent vector field. This gives rise to the second possibility in Theorem 13 .

6.2. The proof of Theorem 16, Let $(M, g, f)$ be a locally homogeneous Lorentzian gradient Ricci soliton of dimension 3.

Case I: Suppose that $(M, g, f)$ is non steady. By Theorem 5 the soliton is rigid.

Case II: Suppose that $(M, g, f)$ is steady. Consequently by Lemma 2, the potential function is a solution of the Eikonal equation $\|\nabla f\|^{2}=\mu$. We distinguish 3 subcases:

Case II-a: $(M, g)$ is steady and $\mu<0$. We apply Theorem 8 to see that $(M, g)$ splits locally as a product and hence the soliton is rigid.

Case II-b: $(M, g)$ is steady and $\mu=0$. We use Theorem 9 to see that the Ricci operator is either 2 or 3 step nilpotent. It follows from work of [1] that there do not exist locally homogeneous 3-dimensional manifolds with 3-step nilpotent Ricci operator. Consequently, the Ricci operator is 2-step nilpotent and $(M, g)$ admits a locally defined parallel null vector field by Theorem 9. Consequently, $(M, g)$ is locally a strict Walker manifold. Consequently, the underlying geometry of $(M, g)$ is given by Theorem 15 the function $f$ is now determined by Theorem 13 
Case II-c: $(M, g)$ is steady and $\mu>0$. Since the scalar curvature is constant, the Ricci operator satisfies $\operatorname{Ric}(\nabla f)=0$, which shows that either $f$ is constant, or otherwise the Ricci operator has a zero eigenvalue. We now consider the different possibilities for the kernel of Ric.

Assume $\operatorname{dim}(\operatorname{ker}\{\operatorname{Ric}\})=1$. It follows from [9] that $(M, g)$ is either a symmetric space or a Lie group. If $(M, g)$ is symmetric, then it is one of the following: a manifold of constant sectional curvature, a product $\mathbb{R} \times N$ where $\left(N, g_{N}\right)$ is of constant curvature, or a three-dimensional Cahen-Wallach symmetric space. Hence, in all the cases, any gradient Ricci soliton is trivial, rigid or the underlying manifold admits a null parallel vector field (and we have already examined that case). Now we concentrate on Lie groups. Since the eigenspaces of the Ricci operator are leftinvariant, since $\nabla f$ has constant norm $\mu>0$, and since $\operatorname{dim}(\operatorname{ker}\{\operatorname{Ric}\})=1$ we have that $\nabla f$ is a left-invariant vector field. Left-invariant Ricci solitons on threedimensional Lorentzian Lie groups were considered in [5], showing that they exist in and only if the Ricci operator has exactly one-single eigenvalue, which must be zero since $\operatorname{Ric}(\nabla f)=0$. This shows that the Ricci operator is three-step nilpotent, but that is not possible due to the analysis carried out in [11].

Finally assume $\operatorname{dim}(\operatorname{ker}\{\operatorname{Ric}\})=2$. In this case the Ricci operator is either diagonalizable or two-step nilpotent. The later implies that the manifold admits locally a null parallel vector field 12 , and again this case has been treated. If the Ricci operator is diagonalizable, then $\|$ Ric $\left\|^{2}= \pm \tau^{2}=\right\| \operatorname{Hess}_{f} \|^{2}$ and Lemma 2(3) shows that $\tau=0$, from where it follows that $(M, g)$ is flat and the soliton is trivial. This completes the proof of Theorem 16.

\section{REFERENCES}

[1] W. Batat, M. Brozos-Vázquez, E. García-Río, and S. Gavino-Fernández, "Ricci solitons on Lorentzian manifolds with large isometry groups", Bull. London Math. Soc. 43 (2011), 12191227.

[2] A. L. Besse, Einstein manifolds, Classics in Mathematics, Springer-Verlag, Berlin, 2008.

[3] L. Berard Bergery and A. Ikemakhen, "On the Holonomy of Lorentzian Manifolds", Proceedings of Symposia in Pure Mathematics 54 (1993), 27-40.

[4] M. Brozos-Vázquez, E. García-Río, and S. Gavino-Fernández, "Locally conformally flat Lorentzian gradient Ricci solitons", J. Geom. Anal. 23 (2013), 1196-1212.

[5] M. Brozos-Vázquez, G. Calvaruso, E. García-Río, and S. Gavino-Fernández, "Threedimensional Lorentzian homogeneous Ricci solitons", Israel J. Math. 188 (2012), 385-403.

[6] M. Brozos-Vázquez, E. García-Río, P. Gilkey, S. Nikčević, and R. Vázquez-Lorenzo, The geometry of Walker manifolds, Synthesis Lectures on Mathematics and Statistics 5, Morgan \& Claypool Publ., Williston, VT, 2009.

[7] M. Cahen and N. Wallach, "Lorentzian symmetric spaces", Bull. Amer. Math. Soc. 76 (1970), $585-591$.

[8] M. Cahen, J. Leroy, M. Parker, F. Tricerri, and L. Vanhecke, "Lorentz manifolds modelled on a Lorentz symmetric space", J. Geom. Phys. 7 (1990), 571-581.

[9] G. Calvaruso, "Homogeneous structures on three-dimensional Lorentzian manifolds, J. Geom. Phys. 57 (2007), 1279-1291.

[10] G. Calvaruso and A. Fino, "Ricci solitons and geometry of four-dimensional non-reductive homogeneous spaces", Canad. J. Math. 64 (2012), 778-804.

[11] G. Calvaruso and O. Kowalski, "On the Ricci operator of locally homogeneous Lorentzian 3-manifolds", Cent. Eur. J. Math. 7 (2009), 124-139.

[12] E. Calviño-Louzao, E. García-Río, E. Vázquez-Abal, and R. Vázquez-Lorenzo, "Curvature operators and generalizations of symmetric spaces in Lorentzian geometry", Adv. Geom. 12 (2012), 83-100.

[13] H.-D. Cao, "Geometry of complete gradient shrinking Ricci solitons", Geometry and analysis. No. 1, 227-246, Adv. Lect. Math. (ALM) 17, Int. Press, Somerville, MA, 2011. 
[14] B. Chow, S.-Ch. Chu, D. Glickenstein, C. Guenther, J. Isenberg, T. Ivey, D. Knopf, P. Lu, F. Luo and L. Ni, The Ricci flow: techniques and applications. Part I. Geometric aspects, Mathematical Surveys and Monographs, 135. American Mathematical Society, Providence, RI, 2007.

[15] M. Eminenti, G. La Nave, and C. Mantegazza, "Ricci solitons: the equation point of view", Manuscripta math. 127 (2008), 345-367.

[16] M. Fernández-López and E. García-Río, "Rigidity of shrinking Ricci solitons", Math. Z. 269 (2011), 461-466.

[17] E. García-Río, P. Gilkey, and S. Nikčević, "Homogeneity of Lorentzian three-manifolds with recurrent curvature", Math. Nachr. 287 (2014), 32-47.

[18] E. García-Río and D. N. Kupeli, "Some splitting theorems for stably causal spacetimes", Gen. Rel. Grav. 30 (1998), 35-44.

[19] S. Helgason, Differential Geometry, Lie groups and symmetric spaces, Pure and Applied Mathematics 80. Academic Press, Inc., New York-London, 1978.

[20] B. Kostant, "Holonomy and the Lie algebra of infinitesimal motions of a Riemannian manifold", Trans. Amer. Math. Soc. 80 (1955), 528-542.

[21] Th. Leistner and P. Nurowski, "Conformal pure radiation with parallel rays", Class. Quantum Grav. 29 (2012), 15pp.

[22] K. Nomizu, "On local and global existence of Killing vector fields", Ann. of Math. 72 (1960), 105-120.

[23] K. Onda, "Lorentz Ricci solitons on 3-dimensional Lie groups", Geom. Dedicata 147 (2010), 313-322.

[24] P. Petersen and W. Wylie, "On gradient Ricci solitons with symmetry", Proc. Amer. Math. Soc. 137 (2009), 2085-2092.

[25] P. Petersen and W. Wylie, "Rigidity of gradient Ricci solitons", Pacific J. Math. 241 (2009), 329-345.

[26] A. Spiro, "A remark on locally homogeneous Riemannian spaces", Result. Math. 24 (1993), $318-325$

MBV: Departmento de Matemáticas, Escola Politécnica Superior, Universidade da CORUÑA, SPAIN

E-mail address: miguel.brozos.vazquez@udc.gal

PG: Mathematics Department, University of Oregon, Eugene OR 97403, USA

E-mail address: gilkey@uoregon.edu

EGR-SGF: Faculty of Mathematics, University of Santiago de Compostela, 15782 Santiago de Compostela, Spain

E-mail address: eduardo.garcia.rio@usc.es sandra.gavino@usc.es 